\title{
KRETSCHMANN INVARIANT AND RELATIONS BETWEEN SPACETIME SINGULARITIES ENTROPY AND INFORMATION
}

\author{
${ }^{1}$ Ioannis Gkigkitzis, ${ }^{2}$ Ioannis Haranas and ${ }^{3}$ Omiros Ragos \\ ${ }^{1}$ Departments of Mathematics, East Carolina University, \\ 124 Austin Building East Fifth Street, Greenville NC 27858-4353, USA \\ ${ }^{2}$ Department of Physics and Astronomy, \\ York University, 4700 Keele Street Toronto, Ontario, M3J 1P3, Canada \\ ${ }^{3}$ Department of Mathematics, Faculty of Sciences, University of Patras, 26500 Patras, Greece
}

Received 2014-02-05; Revised 2014-03-28; Accepted 2014-03-28

\begin{abstract}
Curvature invariants are scalar quantities constructed from tensors that represent curvature. One of the most basic polynomial curvature invariants in general relativity is the Kretschmann scalar. This study is an investigation of this curvature invariant and the connection of geometry to entropy and information of different metrics and black holes. The scalar gives the curvature of the spacetime as a function of the radial distance $r$ in the vicinity as well as inside of the black hole. We derive the Kretschmann Scalar (KS) first for a fifth force metric that incorporates a Yukawa correction, then for a Yukawa type of Schwarzschild black hole, for a Reissner-Nordstrom black hole and finally an internal star metric. Then we investigate the relation and derive the curvature's dependence on the entropy $S$ and number of information N. Finally we discuss the settings in which the entropy's full range of positive and negative values would have a meaningful interpretation. The Kretschmann scalar helps us understand the black hole's appearance as a "whole entity". It can be applied in solar mass size black holes, neutron stars or supermassive black holes at the center of various galaxies.
\end{abstract}

Keywords: Kretschmann Scalar (KS), Schwarzschild Black Hole, Reissner-Nordstrom (RN)

\section{INTRODUCTION}

When we study any space time, it is important above other things to know whether the spacetime is regular or not. By regular spacetime we simply mean that the space time must have regular curvature invariants are finite at all space time points, or contain curvature singularities at which at least one such singularity is infinite. In many cases one of the most useful ways to check that is by checking for the finiteness of the Kretschmann Scalar (from then on KS) which sometimes is also called Riemann tensor squared, in other words Equation 1:

$K=R_{\alpha \beta \gamma \delta} R^{\alpha \beta \gamma \delta}$ where, $R_{\alpha \beta \gamma \delta}$ is the Riemann tensor. In principle the derivation of the $\mathrm{KS}$ is simple, but in practice to actually derive it requires a very long algebraic computation, which can very much be simplified with today's software that perform algebraic and tensorial calculations. In order to calculate the above scalar we first need to calculate the Christoffel symbols of the second kind according to the Equation 2:

$$
I_{\beta \gamma}^{\alpha}=\frac{1}{2} g^{\delta \alpha}\left(\frac{\partial g_{\gamma \delta}}{\partial x^{\beta}}+\frac{\partial g_{\beta \delta}}{\partial x^{\gamma}}+\frac{\partial g_{\gamma \beta}}{\partial x^{\delta}}\right)
$$

Once the Christoffel symbols are calculated we then calculate the Riemann tensor to be Equation 3:

(C) 2014 loannis Gkigkitzis, loannis Haranas and Omiros Ragos. This open access article is distributed under a Creative Commons Attribution (CC-BY) 3.0 license which permits unrestricted use, distribution, and reproduction in any medium, provided the original work is properly credited. DOI: 10.3844/pisp.2014.103.111 


$$
\mathrm{R}_{\beta \gamma \delta}^{\alpha}=\frac{\partial \Gamma_{\beta \delta}^{\alpha}}{\partial \mathrm{x}^{\gamma}}-\frac{\partial \Gamma_{\beta \gamma}^{\alpha}}{\partial \mathrm{x}^{\delta}}+\Gamma_{\beta \delta}^{\mu} \Gamma_{\mu \gamma}^{\alpha}-\Gamma_{\beta \gamma}^{\mu} \Gamma_{\mu \delta}^{\alpha}
$$

For example in a sphere there are only two nonzero Riemann tensor components i.e., $R_{212}^{1}=\sin ^{2} \theta$ and also $R_{221}^{1}=\sin ^{2} \theta$, which exactly characterize the curvature of the sphere. We usually think of the curvature as the Ricci scalar, which can be obtained by contraction of the Riemann tensor, first $R_{\beta \delta}=R_{\beta \alpha \delta}^{\alpha}$ and then $R=R_{\alpha}^{\alpha}$. In the case of a sphere the takes the form (Henry, 2000) Equation 4:

$$
K_{s}=R_{\alpha \beta \gamma \delta} R^{\alpha \beta \gamma \delta}=\frac{4}{a^{4}}
$$

Because it is a sum of squares of tensor components, this is a quadratic invariant. In the case of black holes the calculation of the scalar is required if somebody wants to derive and investigate the curvature of a black hole. The need for calculation of the KS emanates from the fact that in vacuum the field equations of general relativity a zero Gaussian curvature at and in the black hole, thus giving no information about curvature of the spacetime and thus the $K$ scalar need to be computed. In the case of Schwarzschild black holes the KS is (D'inverno, 1992) Equation 5:

$$
K_{b h}=\frac{48 G^{2} M^{2}}{c^{4} r^{6}}
$$

In this contribution we examine the KS of a Yukawa type modified Schwarzschild black holes as it is give in (Haranas and Gkigkitzis, 2013b) and compare this to the Schwarzschild scalar. Furthermore, in an effort to investigate the relation between entropy, information and geometry we write the Yukawa black hole scalar as a function of entropy and information number $N$.

\section{THE YUKAWA POTENTIAL}

Following (Capozziello et al., 2010; Haranas and Gkigkitzis, 2013b) we say that theories derived from the action:

$$
A=\int_{v} \sqrt{-g}\left(f\left(R, \nabla R, \nabla^{2} R, \nabla^{k} R, \phi\right)-\frac{\varepsilon}{2} g^{\mu v} \phi_{; \mu} \phi_{; \nu}+L_{m}\right) d^{4} x
$$

Result to Yukawa corrections to the gravitational potential where $f(R)$ is an analytic function of Ricci scalar, $g$ is the determinant of the metric $g_{\mu v}$ and $L m$ is a fluid-matter Lagrangean and where $f$ is an unspecified function of curvature invariants $\mathrm{R}$ and $\nabla R$ and of scalar fields $\phi$. Actually, more complicated invariants like $R^{\mu v} R_{\mu v}$ and $R^{\mu \nu \alpha \beta} R_{\mu \nu \alpha \beta}$ and $C^{\mu v \alpha \beta} C_{\mu \nu \alpha \beta}$. In the weak field limit theories described by Equation 6 result to potentials of the form (Capozziello et al., 2010) Equation 7:

$$
V(r)=-\frac{G_{\infty} M}{r}\left[1+\sum_{k=1}^{n} \alpha_{k} e^{\frac{r}{\lambda_{k}}}\right]
$$

where, $G_{\infty}$ is the value of the gravitational constant as measured at infinity, $\lambda_{k}$ is the interaction length of the $k^{\text {th }}$ component of non-Newtonian corrections and $\alpha_{k}$ amplitude of is term is normalized to the standard.

Newtonian term. If somebody considers the first term in the series of Equation 9, we obtain the following potential Equation 8:

$V(r)=-\frac{G_{\infty} M}{r}\left[1+\alpha_{1} e^{\frac{r}{\lambda_{1}}}\right]$

The second term is a Yukawa type of correction to the Newtonian potential and its effect can be parameterized by $\mathrm{a}_{1}$ and $\lambda_{l}$ which for simplicity we will call $\alpha$ and $\lambda$. For large distances, i.e., $r>>\lambda$ the exponential term vanishes and the gravitational constant is simply $G_{\infty}$. If $r<<\lambda$ the exponential becomes unity.

\section{THE METRIC AND RICCI TENSOR AND THE KRETSCHMANN SCALAR}

Writing the metric of a spherically symmetric star in the following way:

$$
d s^{2}=c^{2} e^{2 \mu(r, t)} d t^{2}-e^{2 v(r, t)} d r^{2}-e^{2 \xi(r, t)} d \Omega^{2}
$$

where, $\mu, v, \xi$ are in general functions of radial distance $r$ and time $t$ and $d \Omega^{2}=d \theta^{2}+\sin ^{2} \theta d \phi^{2}$. For a static metric in Equation 9 the Ricci tensor is diagonal and therefore we obtain the following nonzero components Equation 10 to 13:

$$
\begin{aligned}
& R_{0}^{0}=e^{-2 \mu}\left(2 \ddot{\xi}+\ddot{v}+2 \dot{\xi}^{2}+\dot{v}^{2}-\dot{\mu}(2 \dot{\xi}+\dot{v})\right) \\
& -e^{-2 v}\left(\mu^{\prime \prime}+\mu^{\prime}\left(2 \xi^{\prime}+\mu^{\prime} v^{\prime}\right)\right) \\
& R_{1}^{1}=e^{-2 \mu}(\ddot{v}+\dot{v}(2 \dot{\xi}-\dot{\mu}+\dot{v})) \\
& -e^{-2 v}\left(2 \xi^{\prime 2}+\mu^{2}-v^{\prime}\left(2 \xi^{\prime}+\mu^{\prime}\right)\right) \\
& R_{2}^{2}=R_{3}^{3}=e^{-2 \xi}+e^{-2 \mu}(\ddot{\xi}+\dot{\xi}(2 \dot{\xi}-\dot{\mu}+\dot{v})) \\
& -e^{-2 v}\left(\xi^{\prime \prime}+\xi^{\prime}\left(2 \xi^{\prime}+\mu^{\prime}-v^{\prime}\right)\right)
\end{aligned}
$$




$$
R_{01}=2\left(\dot{\xi}^{\prime}+\xi^{\prime}(\dot{\xi}-\dot{v})-\mu^{\prime} \dot{\xi}\right)
$$

Similarly, we find that Equation 15 to 17:

$$
\begin{aligned}
& K_{1}=-R_{01}^{01}=e^{-(\mu+v)} \frac{d}{d r}\left(\frac{d \mu}{d r} e^{\mu-v}\right) \\
& K_{2}=-R_{02}^{02}=-R_{03}^{03}=e^{-(2 v)} \frac{d \xi}{d r} \frac{d \mu}{d r} \\
& K_{2}=-R_{12}^{12}=-R_{13}^{13}=e^{-(v+\xi)} \frac{d}{d r}\left(e^{\xi-v} \frac{d \xi}{d r}\right) \\
& K_{4}=-R_{23}^{23}=e^{-(2 \xi)}+e^{-(2 v)}\left(\frac{d \xi}{d r}\right)^{2}
\end{aligned}
$$

And therefore the KS becomes:

$$
K=4\left(K_{1}\right)^{2}+8\left(K_{2}\right)^{2}+8\left(K_{3}\right)^{2}+4\left(K_{4}\right)^{2}
$$

Next in our effort to investigate KS singularities of various metric let us now proceed with a fifth force metric that incorporates a Yukawa correction as it is given by (Spallicci, 1991) curved only in the time coordinate and used for measurements in the solar system experiments namely:

$d s^{2}=c^{2}\left[1-\frac{2 G M}{c^{2} r}\left(1+\alpha e^{-r / \lambda}\right)\right] d t^{2}-d r^{2}-r^{2} d \Omega^{2}$

And therefore Equation 19 to 21:

$v(r)=0$

$\xi(r)=r$

$\mu(r)\left(r=\frac{1}{2} \ln \left[\left[1-\frac{2 G M}{c^{2} r}\left(1+\alpha e^{-\frac{r}{\lambda}}\right)\right]\right]\right)$

Omitting orders $O\left(c^{-8}\right), O\left(c^{-6}\right)$ and $e^{-3 r / \lambda}$ and $e^{-4 r / \lambda}$ the final expression for the KS becomes Equation 22:

$$
K \cong \frac{48 G^{2} M^{2}}{c^{4} r^{6} T^{2}}\left[\begin{array}{l}
\frac{1}{2}+\alpha e^{-\frac{r}{\lambda}}\left(1+\frac{r}{\lambda}+\frac{r^{2}}{3 \lambda^{2}}\right) \\
+\alpha^{2} e^{-\frac{2 r}{\lambda}}\left(\frac{1}{2}+\frac{r}{\lambda}+\frac{5 r^{2}}{6 \lambda^{2}}+\frac{r^{3}}{3 \lambda^{3}}+\frac{r^{4}}{12 \lambda^{4}}\right)
\end{array}\right]
$$

where, Equation 23:

$$
T=\left[1-\frac{2 G M}{c^{2} r}\left(1+\alpha e^{-\frac{r}{\lambda}}\right)\right]
$$

For a black hole of mass $M$ the KS possesses an essential singularity at the value of $r=r_{H}$ where $T=0$ given by Equation 24:

$r_{H}=\frac{2 G M}{c^{2}}+\lambda W\left(\frac{2 G M \alpha}{\lambda c^{2}} e^{\frac{2 G M}{\lambda c^{2}}}\right)$

where, $W$ is the Lambert function of the indicated argument. Next, following (Haranas and Gkigkitzis, 2013a) we write the metric of a Yukawa type of Schwarzschild black hole that is curved in along both the time and radial coordinate, according to the equation Equation 25:

$$
\begin{aligned}
& d s^{2}=c^{2}\left[1-\frac{2 G M}{c^{2} r}\left(1+\alpha e^{-r / \lambda}\right)\right] d t^{2} \\
& -\left[1-\frac{2 G M}{c^{2} r}\left(1+\alpha e^{-r / \lambda}\right)\right]^{-1} d r^{2}-r^{2} d \Omega^{2}
\end{aligned}
$$

where, $d \Omega^{2}=d \theta^{2}+\sin ^{2} \theta d \phi^{2}$ and upon comparing Equation 9 and 14 we obtain that Equation 26 to 28 :

$$
\begin{aligned}
& v(r)=\frac{1}{2} \ln \left[\left[1-\frac{2 G M}{c^{2} r}\left(1+\alpha e^{-\frac{r}{\lambda}}\right)\right]^{-1}\right] \\
& \xi(r)=r \\
& \mu(r)=\frac{1}{2} \ln \left[\left[1-\frac{2 G M}{c^{2} r}\left(1+\alpha e^{-\frac{r}{\lambda}}\right)\right]\right]
\end{aligned}
$$

Therefore using Equation 18 the final expression for the KS becomes:

$$
\begin{aligned}
& K_{Y u k}=K_{s c h}+K_{c o r}=\frac{48 G^{2} M^{2}}{c^{4} r^{6}} \\
& {\left[\begin{array}{l}
1+\alpha e^{-\frac{r}{\lambda}}\left(1+\frac{16 r}{12 \lambda}+\frac{r^{2}}{\lambda^{2}}\right) \\
+\alpha^{2} e^{-\frac{2 r}{\lambda}}\left(1+\frac{16 r}{12 \lambda}+\frac{r^{2}}{\lambda^{2}}+\frac{r^{3}}{3 \lambda^{3}}+\frac{r^{4}}{12 \lambda^{4}}\right)
\end{array}\right]}
\end{aligned}
$$


The KS equation above Equation 28 does not possess an essential singularity. This could be due to the fact that the metric elements $g_{\mu \nu}$ cancels with the corresponding $g_{\mu \nu}^{-1}$. As a check we see that when $\alpha=0$ we immediately obtain the KS of a Schwarzschild metric as it is given in (Henry, 2000).

Next, we use the non-rotating but electrically charged source vacuum solution as it is given by the ReissnerNordstrom solution (Misner and Wheeler, 1973):

$$
\begin{aligned}
& d s^{2}=c^{2}\left[1-\frac{2 G M}{c^{2} r}+\frac{G Q^{2}}{4 \pi \varepsilon_{0} c^{4} r^{2}}\right] d t^{2} \\
& -\left[1-\frac{2 G M}{c^{2} r}+\frac{G Q^{2}}{4 \pi \varepsilon_{0} c^{4} r^{2}}\right]^{-1} d r^{2}-r^{2} d \Omega^{2}
\end{aligned}
$$

The final expression for the KS becomes:

$$
K_{R N}=\frac{48 G^{2} M^{2}}{c^{4} r^{6}}\left(1+\frac{2 Q^{2}}{c^{2} r M}+\frac{7 Q^{4}}{48 c^{4} r^{2} M^{2}}\right)
$$

As a check we see that when $Q=0$ we immediately obtain the KS of a Schwarzschild metric as it is given in (Henry, 2000). Similarly, the KS equation of the ReissnerNordstrom above Equation 29 does not possess an essential singularity but only the $r=0$ one. Moreover, let us consider the internal star metric solution that reads (Stephani, 1990) Equation 32:

$$
\begin{aligned}
& d s^{2}=c^{2}\left[\frac{3}{2} \sqrt{1-\frac{2 G M}{R c^{2}}-\frac{1}{2}} \sqrt{1-\frac{2 G M r^{2}}{R c^{2}}}\right]^{2} \\
& d t^{2}-\left[1-\frac{2 G M r^{2}}{c^{2} R^{3}}\right]^{-1} d r^{2}-r^{2} d \Omega^{2}
\end{aligned}
$$

The final expression for the KS becomes:

$$
\begin{aligned}
& K_{\mathrm{int}}=\frac{48 G^{2} M^{2}}{c^{4} R^{6}}+\frac{48 G^{2} M^{2}}{c^{4} R^{6}\left(\sqrt{1-\frac{2 G M r^{2}}{R c^{2}}}-3 \sqrt{1-\frac{2 G M r^{2}}{R c^{2}}}\right)^{2}} \\
& -\frac{96 G^{3} M^{3} r^{2}}{c^{6} R^{9}\left(\sqrt{1-\frac{2 G M r^{2}}{R c^{2}}}-3 \sqrt{1-\frac{2 G M r^{2}}{R c^{2}}}\right)^{2}}
\end{aligned}
$$

The KS equation i.e., Equation 33 possesses an essential singularity at the following values of $r$ :

$$
r_{K \mathrm{int}}= \pm R \sqrt{\frac{9 G M-4 c^{2} R}{G M}}
$$

Furthermore, Equation 33 approaches the KS of the Schwarzschild KS if the two RHS terms become zero for the values of $r$ given by:

$$
r_{\text {int }}= \pm c \sqrt{2 \frac{R^{3}}{G M}}
$$

\section{KRETSCHMANN SCALAR AND IT RELATION TO ENTROPY AND NUMBER INFORMATION}

In general relativity there exist a set of curvature invariants that they are scalars. They can be formed from the Riemann, Ricci and Weyl tensors respectively and they describe various possible operations such that, covariant differentiation, contraction. We can obtain various invariants that they are formed from these curvature tensors that play an important role in the classification of spacetimes. Invariants useful in distinguishing Riemannian manifolds or manifolds that they have a positive and well defined metric tensor. In order to investigate true singularities one must look at quantities that are independent of the choice of coordinates. One of these is the KS which, at the origin only or $r=0$ possesses a singularity that is a physical singularity which we can also call gravitational singularity. At $r=0$ the curvature becomes infinite indicating the presence of a singularity. At this point the metric and space-time itself, is no longer well-defined. For a long time it was thought that such a solution was non-physical. However, a greater understanding of general relativity led to the realization that such singularities were a generic feature of the theory and not just an exotic special case. Such solutions are now believed to exist as called black holes.

This contribution is an effort to connect geometry to information and an attempt to investigate the consequences of essential KS entropy and information singularities that may result from the elimination of mass $\mathrm{M}$ that enters the metric. Since $\alpha=3.04 \times 10^{-8}$ and $r<\lambda=$ $4.94 \times 10^{15} \mathrm{~m}$ (Haranas and Gkigkitzis, 2011) and considering a 1 solar mass black hole Equation 29 the exponential quantities $e^{-r / \lambda} \cong 1$ and $e^{-2 r / \lambda} \cong 1$ are both approximately equal Equation 22 simplifies to:

$$
K \cong \frac{48 G^{2} M^{2}}{c^{4} r^{6}\left[1-\frac{2 G M}{r c^{2}}(1+\alpha)\right]^{2}}\left[\begin{array}{l}
\frac{1}{2}+\alpha\left(1+\frac{r}{\lambda}\right) \\
+\alpha^{2}\left(\frac{1}{2}+\frac{r}{\lambda}\right)
\end{array}\right]
$$


Therefore we find that the KS has an essential singularity that is given by the equation:

$$
r_{K S_{H}} \cong \frac{2 G M}{c^{2}}(1+\alpha)=R_{S c h}(1+\alpha)
$$

where, $R_{\text {Sch }}$ is the gravitational radius of the Schwarzschild black hole. At this point we see that this particular metric does posses an event horizon and therefore the entropy cannot be calculated on the horizon. However, the existence of a singular boundary is apparent: the "Kretcmann horizon" given by Equation 37. To proceed let us assign a definition of entropy, the $\mathrm{KS}$ entropy, similar to that calculated to (Haranas and Gkigkitzis, 2011), which reads as follows:

$$
\begin{aligned}
S_{K S} & =\left(\frac{c^{3} k_{B}}{4 G \hbar}\right) A_{K S}=\frac{4 \pi(1+\alpha)^{2} k_{B} G}{c \hbar} \\
M^{2} & =4 \pi(1+\alpha)^{2} k_{B}\left(\frac{M}{m_{p}}\right)^{2}
\end{aligned}
$$

where, $A_{K S}$ is the surface area defined by the Equation 37. This definition suggests that the KS horizon is a depository of information (or infinite information). Next we obtain that Equation 40:

$$
M=\frac{m_{p}}{2(1+\alpha)} \sqrt{\frac{S_{K S}}{\pi k_{B}}}
$$

And:

$$
r_{K S_{H}}=\frac{G m_{p}}{c^{2}(1+\alpha)} \sqrt{\frac{S_{K S}}{\pi k_{B}}}
$$

Substituting Equation 38 in Equation 39 in Equation 36 we express $\mathrm{KS}$ as a function of the entropy $S_{K S}$ and we find that:

$$
\begin{aligned}
& K_{K S}\left(S_{K S}\right)=\frac{12 c^{8} k_{B}}{\alpha^{2} G^{4} m_{p}^{2} S_{K S}^{2}}\left[\frac{1}{2}+\frac{G m_{p}}{c^{2} \lambda(1+\alpha)} \sqrt{\frac{S_{K S}}{k_{B}}}\right] \\
& +\alpha^{2}\left(1+\frac{G m_{P}}{c^{2} \lambda(1+\alpha)} \sqrt{\frac{S_{K S}}{k_{B}}}\right)
\end{aligned}
$$

For 1 solar mass black hole the most significant contribution comes from the coefficient of the $1 / \mathrm{S}^{2}$ term and therefore the KS entropy can be written as Equation 42 :

$$
\left(S_{K S}\right)= \pm \frac{c^{4} k_{B}(1+\alpha)^{3}}{\alpha G^{2} m_{p}^{2}} \sqrt{\frac{6}{k_{K S}}}
$$

And therefore the number of information $N$ can be written as a function of the KS in the following way:

$$
N\left(K_{K S}\right) \cong \frac{c^{4}(1+\alpha)^{3}}{\alpha G^{2} m_{p}^{2} \ln 2} \sqrt{\frac{6}{k_{K S}}}
$$

Similarly, taking into account the same assumptions as in Equation 35 we obtain KS of a fully curved Yukawa type of metric is given by Equation 28 can be approximated in the following way Equation 44:

$$
\begin{aligned}
& K_{Y u k}=K_{s c h}+K_{c o r} \cong \frac{48 G^{2} M^{2}}{c^{4} r^{6}} \\
& {\left[1+\alpha\left(1+\frac{16 r}{12 \lambda}\right)+\alpha^{2}\left(1+\frac{16 r}{12 \lambda}\right)\right]}
\end{aligned}
$$

Next, $u$ sing expressions for the mass and the gravitational radius for a Yukawa black hole as a functions of entropy for a solar mass black hole, as it given in (Haranas and Gkigkitzis, 2013b) we are trying to express the singularity in the gravitational radius as a singularity in essence in entropy S Equation 45 and 46:

$$
\begin{aligned}
& M(S)=\frac{m_{p}}{2} \sqrt{\frac{S}{\pi k_{B}(1-\alpha)}} \\
& r_{H}=\frac{2 G M}{c^{2}}(1+\alpha) \\
& =\frac{2 G}{c^{2}}(1+\alpha) \frac{m_{p}}{2} \sqrt{\frac{(1+\alpha) S}{\pi k_{B}}} \\
& =\ell p(1+\alpha) \sqrt{\frac{S}{\pi k_{B}(1+\alpha)}}
\end{aligned}
$$

Equation 41 has only one singularity at $r=0$ and there is no other essential singularity. Using Equation 41 and taking into account the same assumptions as in Equation 35 we obtain that the KS as a function of entropy $S$ on the horizon of such a black hole up to the $O\left(\alpha^{3}\right)$ the most significant contribution comes from the coefficient of the $1 / S^{2}$ term and therefore the KS can be written as Equation 47:

$$
K_{\text {Yuk }} \cong \frac{12 \pi^{2} G^{2} m_{p}^{2} k_{B}^{2}}{c^{4} \ell_{p}^{6}(1+\alpha)^{6} S^{2}}\left(1-\alpha-\alpha^{3}\right)
$$


Which tends to zero as the entropy approaches infinity. Solving for the entropy $\mathrm{S}$ we obtain an expression of the entropy as a function of the scalar $K_{y u k}$ to be Equation 48:

$$
S= \pm \frac{2 \pi G m_{p} k_{B}}{c^{2} \ell_{p}^{3}} \frac{(1-\alpha)}{(1+\alpha)^{3}}\left[\frac{3\left(1+\alpha+\alpha^{2}\right)}{K_{y u k}}\right]^{1 / 2}
$$

Next using (Haranas and Gkigkitzis, 2013a) we write the entropy to be: $S=N k_{B} l n 2$, we write the $\mathrm{KS}$ as a function of entropy and number of information $\mathrm{N}$ according to the equations:

$$
N=\frac{2 \pi G m_{p}}{\ln 2 c^{2} \ell_{p}^{3}} \frac{(1-\alpha)}{(1+\alpha)^{3}}\left[\frac{3\left(1+\alpha+\alpha^{2}\right)}{K_{Y u k}}\right]^{1 / 2}
$$

$N=0$ only if alpha imaginary. Both Equation 43 and 44 can exhibit a singularity if and only if the coupling constant becomes negative i.e., $\alpha=-1$. A negative $\alpha$ would introduce a repulsive component in the Yukawa correction something that it is known as a fifth force.

Next we consider the Reissner-Nordstrom (RN) metric the KS does not possess a singularity besides the $r$ $=0$ case. The event horizon RN metric has the following solutions Equation 50:

$$
r_{R N_{H}}=\frac{G M}{c^{2}} \pm \frac{1}{2 \varepsilon_{0} c^{2}} \sqrt{\frac{G \varepsilon_{0}\left(Q^{2}+4 \pi \varepsilon_{0} G M^{2}\right)}{\pi}}
$$

And therefore the corresponding entropy becomes Equation 51:

$$
\begin{aligned}
& S_{R N}=\left(\frac{c^{3} k_{\mathbf{B}}}{4 G \hbar}\right) A_{R N}=\frac{2 \pi k_{B} G M^{2}}{c \hbar} \\
& +\frac{k_{B} Q^{2}}{4 \varepsilon_{0} c \hbar} \pm \frac{k_{B} M \sqrt{\pi G \varepsilon_{0}\left(Q^{2}+4 \varepsilon_{0} G M^{2}\right)}}{\varepsilon_{0} c \hbar}
\end{aligned}
$$

Solving for the mass $\mathrm{M}$ in terms of the entropy we obtain Equation 52:

$$
M \frac{1}{8} \sqrt{\frac{16 \hbar S}{\pi G k_{B}}+\frac{k_{B} Q^{4}}{\pi \varepsilon_{0}^{2} c \hbar G S}-\frac{8 Q^{2}}{\pi \varepsilon_{0} G}}
$$

Similarly the event horizon as a function of entropy becomes Equation 53:

$$
\begin{aligned}
& r_{N R_{H}}=\frac{1}{8 c^{2}} \sqrt{\frac{16 G \hbar S}{\pi k_{B}}+\frac{G k_{B} Q^{4}}{\pi \varepsilon_{0}^{2} c \hbar S}-\frac{8 G Q^{2}}{\pi \varepsilon_{0}}} \\
& \pm \frac{1}{2 \varepsilon c^{2}} \sqrt{\frac{\varepsilon_{0}}{\pi}\left(G Q^{2}+\frac{c \hbar G S \varepsilon_{0}}{k_{B}}+\frac{k_{B} G Q^{4}}{16 c \hbar \varepsilon_{0} S}-\frac{G Q^{2}}{2 \varepsilon_{0}}\right)}
\end{aligned}
$$

And therefore substituting for $r_{N R H}$ and $M$ we write the $\mathrm{KS}$ of the Reissner-Nordstrom (RN) metric as a function of entropy in the following way Equation 54 and 55:

$$
\begin{aligned}
& K_{K S}=\frac{7 G^{2} Q^{4}}{2 \pi^{2} c^{8} \varepsilon_{0}^{2} \Phi^{8}}+\frac{3 Q^{2}}{c^{6} \varepsilon_{0} \Phi^{7}} \\
& \sqrt{\frac{16 c \hbar G^{3} S}{\pi^{3} k_{B}}+\frac{k_{B}^{2} G^{3} Q^{4}}{c \hbar \varepsilon_{0}^{2} k_{B} \pi^{3} S}-\frac{8 G^{3} Q^{2}}{\varepsilon_{0} \pi^{3}}} \\
& +\frac{3}{4 \pi k_{B} c^{4} \Phi^{6}}\left(16 G c \hbar S+\frac{k_{B}^{2} G Q^{4}}{c \hbar \varepsilon_{0}^{2} S}-\frac{8 k_{B} G Q^{2}}{\varepsilon_{0}}\right)
\end{aligned}
$$

Where:

$$
\begin{gathered}
\Phi=\frac{1}{8 c^{2}} \sqrt{\frac{16 c \hbar G S^{2}+\frac{k_{B}^{2} Q^{4}}{c \hbar \varepsilon_{0}^{2}}-\frac{8 k_{B} Q^{2} S}{\varepsilon_{0}}}{\pi k_{B} S}}+\frac{1}{2 \varepsilon_{0} c^{2}} \\
\sqrt{\frac{\varepsilon_{0} G}{\pi}\left(Q^{2}+\frac{\varepsilon_{0}}{16 k_{B} S}\left(16 c \hbar S^{2}+\frac{k_{B}^{2} Q^{2}}{c \hbar \varepsilon_{0}^{2}}-\frac{8 k_{B} Q^{2} S}{\varepsilon_{0}}\right)\right)}
\end{gathered}
$$

The KS of the Reissner-Nordstrom (RN) metric possesses a singularity when $\Phi=0$, for the following values of entropy out of which the first two are real numbers Equation 56 to 59:

$$
\begin{aligned}
& S_{1}=\frac{k_{B} Q^{2}\left(2+c \sqrt{\varepsilon_{0}}\right)}{4 c \hbar \varepsilon_{0}\left(2+c \varepsilon_{0}\right)} \\
& S_{2}=\frac{k_{B} Q^{2}\left(2-c \sqrt{\varepsilon_{0}}\right)}{4 c \hbar \varepsilon_{0}\left(2+c \varepsilon_{0}\right)} \text { SOS -ve } \\
& S_{3}=\frac{k_{B} Q^{2}\left(2 i+c \sqrt{\varepsilon_{0}}\right)}{4 c \hbar \varepsilon_{0}\left(2 i-c \varepsilon_{0}\right)} \\
& S_{4}=\frac{k_{B} Q^{2}\left(2 i-c \sqrt{\varepsilon_{0}}\right)}{4 c \hbar \varepsilon_{0}\left(2 i+c \varepsilon_{0}\right)}
\end{aligned}
$$

And therefore the values of the number of information $N$ at which Equation 49 is singular are Equation 60 to 63 : 
$N_{1}=\frac{Q^{2}\left(2+c \sqrt{\varepsilon_{0}}\right)}{4 c \hbar \varepsilon_{0}\left(2-c \varepsilon_{0}\right) \ln 2}$

$N_{2}=\frac{Q^{2}\left(2-c \sqrt{\varepsilon_{0}}\right)}{4 c \hbar \varepsilon_{0}\left(2+c \varepsilon_{0}\right) \ln 2}$

$N_{3}=\frac{Q^{2}\left(2 i+c \sqrt{\varepsilon_{0}}\right)}{4 c \hbar \varepsilon_{0}\left(2 i-c \varepsilon_{0}\right) \ln 2}$

$N_{4}=\frac{Q^{2}\left(2 i-c \sqrt{\varepsilon_{0}}\right)}{4 c \hbar \varepsilon_{0}\left(2 i+c \varepsilon_{0}\right) \ln 2}$

Finally for the internal metric solution of a star there is a metric singularity at $r=r_{H}$ when Equation 64:

$r_{H}= \pm c \sqrt{\frac{R^{3}}{2 G M}}$

And therefore the entropy becomes Equation 65:

$S_{K S}=\left(\frac{c^{3} k_{B}}{4 G \hbar}\right) A_{K S}=\frac{\pi k_{B} c^{5}}{2 G^{2} M \hbar} R^{3}=\frac{\pi k_{B}}{\ell_{p}^{2}}\left(\frac{R^{3}}{R_{g^{\prime} \times h}}\right)$

where, $R_{\text {grsch }}$ is the Schwarzschild gravitational radius. Therefore solving for the mass $M$ from Equation 66 we obtain that:

$M=\frac{\pi k_{B}}{\ell_{p}^{2}}\left(\frac{c^{2} R^{3}}{G S}\right)$

And thus Equation 33 of the corresponding KS can be written in terms of the entropy $\mathrm{S}$ as follows Equation 67:

$$
\begin{aligned}
& K_{\text {int }}=\frac{12 \pi^{2} c^{6} k_{B}^{2}}{G^{2} \hbar^{2} S^{2}} \\
& +\frac{12 \pi^{2} c^{6} k_{B}^{2}}{G^{2} \hbar^{2} S^{2}\left(\sqrt{1-\frac{\pi k_{B} c^{3} R^{2}}{G \hbar S}}-3 \sqrt{1-\frac{\pi k_{B} c^{3} r^{2}}{G \hbar S}}\right)^{2}} \\
& -\frac{12 \pi^{3} c^{9} k_{B}^{3} r^{2}}{G^{3} \hbar^{3} S^{3}\left(\sqrt{1-\frac{\pi k_{B} c^{3} r^{2}}{G \hbar S}}-3 \sqrt{1-\frac{\pi k_{B} c^{3} R^{2}}{G \hbar S}}\right)}
\end{aligned}
$$

The KS of the internal metric solution has an essential entropy singularity that is given from Equation 68:

$$
S_{K S_{\text {ivt }}}=\frac{\pi k_{B} c^{3}}{8 G \hbar}\left(9 R^{2}-r^{2}\right)
$$

Or an equivalent number of information singularity that takes the value Equation 69:

$$
N_{K S_{\text {itt }}}=\frac{\pi c^{3}}{8 G \hbar \ln 2}\left(9 R^{2}-r^{2}\right)
$$

To compare we write the KS of the Schwarzschild metric on the horizon an a function of entropy and information to be Equation 70:

$$
K_{S c h}=\frac{12 G \hbar}{\pi c^{3} k_{B} r^{6}} S=\frac{12}{\pi k_{B}}\left(\frac{\ell_{p}}{r^{3}}\right)^{2} S
$$

\section{DISCUSSION}

The Schwarzschild solution was the first physically significant solution of the field equations of general relativity. It showed how space time is curved around spherically symmetric distribution of matter. The KS for a Schwarzschild black hole is inversely proportional to the distance. In Equation 18 a formula for the KS is given for a general spherically symmetric metric which we apply to a fifth force metric that incorporates a Yukawa correction and notice that KS possesses an essential singularity at the horizon. Then we write the metric of a Yukawa type of Schwarzschild black hole that is curved in along both the time and radial coordinate and we notice in Equation 29 that the singularity disappears. This is also the case for the nonrotating but electrically charged source vacuum solution as it is given by the Reissner-Nordstrom solution in Equation 29-31. In considering the internal star metric solution we see again the occurrence of an essential singularity in Equation 34. The introduction of the concept of entropy leads to the definition of "KS horizon", beyond the distinction between gravitational singularities and essential geometric singularities. This allows us to define and investigate singularities in essence in entropy S. The equations for the entropy, such as $42,48,50,58$ and 59 as well as the equations for the corresponding information may result in negative and complex values for the entropy, which makes it necessary to seek an appropriate framework for the interpretation of these results in an intersection between or combination of general relativity and quantum mechanics. 
The scalar gives the curvature of the spacetime as a function of the radial distance $r$ in the vicinity as well as inside of the black hole. Furthermore, the Kretschmann scalar helps us understand the black hole's appearance as a whole entity from a geometric as physical point of view. It can be applied in solar mass size black holes, neutron stars or supermassive black holes at the center of various galaxies.

Finding a setting in which the entropy's full range of values would have a meaningful interpretation is an essential step in interpreting our results on entropy. Negative entropy appears as an interesting feature of higher derivative gravity (Cvetic et al., 2002) (Schwarzschild-Anti-deSitter and Schwarzschild-deSitter gravity black holes) and has been suggested that this appearance of negative entropy may indicate a new type instability where a transition between SdS (SAdS) black holes with negative entropy to SAdS (SdS) black holes with positive entropy would occur and where the classical thermodynamics would not apply any more. It was also suggested that such a new type phase transition is presumably related with the known fact that strong energy condition in higher derivative gravity maybe violated (Cvetic et al., 2002). On the other hand, entropy can be negative and erasing bit from a system can result in a net gain of work, leading to the result of the effect that a quantum computer may cool itself by erasing bits of information (Del Rio et al., 2011). It will be interesting if such negative entropy idea can apply to black holes. This can change Hawking radiation-maybe under some condition, the black hole can also be cooling system (not only radiating) or other unknown as of now effects may be happen. Modeling our universe as just a big computer, deleting information in a black hole may occur through accretion matter to black hole and the black hole does not radiate but cools the universe. This seems to be in accordance with another approach of suggesting that Hawking radiation, via quantum tunneling, carries entropy out of the black hole, a process that represents a net gain of information drawn out from the black hole (Zhang et al., 2011). So, Hawking radiation carries not only entropy, but also information, out of the black hole.

Negative values for the entropy point towards a quantum entropy which can be understood operationally as quantum channel capacity (Horodecki et al., 2005). In the language of communication theory, the amount of information originating from a source is the memory required to faithfully represent its output and this amount is given by its entropy. Negative entropy has a physical interpretation in terms of how much quantum communication is needed to gain complete quantum information possession of a system in the total state (Horodecki et al., 2005). It is possible for a black hole to correlate with its environment in ways such as quantum entanglement using procedures such as quantum teleportation where qubits (the basic units of quantum information) can be transmitted exactly (in principle) from one location to another, without the qubits being transmitted through the intervening space. Therefor it can be correlated to a reference system that regulates maximally entangled states of a system which can be used as a teleportation protocol to transmit quantum states from a source to a receiver.

Landauer's principle states that the erasure of data stored in a system has an inherent work cost and therefore dissipates heat. However, this consideration assumes that the information about the system to be erased is classical and does not extend to the general case where an observer may have quantum information about the system to be erased, for instance by means of a quantum memory entangled with the system. The study cost of erasure is determined by the entropy of the system, conditioned on the quantum information an observer has about it. The more an observer knows about the system, the less it costs to erase it. Entropies can become negative in the quantum case, when an observer who is strongly correlated with a system may gain work while erasing it, thereby cooling the environment (Del Rio et al., 2011).

The notion of complex-valued as well as negative information entropy remains to be given some physical sense interpretation through possibly an interaction range consideration. We can only conjecture that the complex entropy may be the result of a certain background free energy and/or scale invariance. From a quantum mechanics point of view, this might indicate the presence of a non Hermitian Hamiltonian (Rotundo and Ausloos, 2013). Certain observations indicate this direction. An attempt to a dynamical interpretation of a classical complex free energy is found in (Zwerger, 1985) where the author pointed out that the problem is to determine a characteristic "relaxation" time for some process in which a dynamical equation (Langevin or FokkerPlanck) is connected to some Hamiltonian or some corresponding transfer matrix. A probability current can be written, in fact, in terms of some unstable mode times an equilibrium factor which is the imaginary part of the free energy. The imaginary part of the free energy (or largest eigenvalues) give some information about the "nucleation stage" of the dynamics, i.e., an initial decay rate out of a metastable state of a system after a sudden change in an effective field (Zwerger, 1985). This metastable state in our may correspond to a physical 
gravitational singularity, independent of coordinates, where instead of "relaxation time", one may consider the "spatial aspect of the phenomenon", e.g., through some correlation range length $\xi$ that corresponds to a certain differential distance of the horizon. The real part, of course of such a free energy, determines the equilibrium energy state.

\section{CONCLUSION}

Invariance plays a very essential role in mathematics and physics. It is one of most important tools of tensor analysis as well as of the theory of relativity and cosmology. Black hole is perhaps the least exemplary of physical entities. Indeed, despite their appearance at the center of most if not all spiral galaxies, black holes are entities for which it is most often said "physics breaks down". In addition to this, the physics of black holes has been revolutionized by developments that grew out of Jacob Bekenstein's realization that black holes have entropy. Steven Hawking raised profound issues concerning the loss of information in black hole evaporation and the consistency of quantum mechanics in a world with gravity. In this direction, we have studied Kretschmann scalar curvature invariant which may be of great utility in the study and classification of black hole singularities. We have discussed the notion of negative and complex-valued information entropy for which there is a need to give some physical sense interpretation through possibly quantum considerations. These new findings may open the door to solving many previously intractable problems in quantum information theory of black holes. It is concluded that such generalizations are not only interesting and necessary for discussing information theoretic properties of black hole gravity, but also may give new insight into conceptual ideas about entropy and information.

\section{REFERENCES}

Capozziello, S., G. Cristofano and M. De Laurentis, 2010. Astrophysical structures from primordial quantum black holes. Eur. Phys. J. C, 69: 293-303. DOI: 10.1140/epjc/s10052-010-1387-2

Cvetic, M., S. Nojiri and S.D. Odintsov, 2002. Black hole thermodynamics and negative entropy in desitter and anti-desitter einstein-gauss-bonnet gravity. Nucl. Phys. B. 628: 295-330. DOI: 10.1016/S0550-3213(02)00075-5

D'inverno, R., 1992. Introducting einstein's relativity. Am. J. Phys., 65: 897-897.
Del Rio, L., J. Aberg, R. Renner, O. Dahlsten and V. Vedral et al., 2011. The thermodynamic meaning of negative entropy. Nature, 476: 476-476. DOI: 10.1038 /nature 10123

Haranas, I. and I. Gkigkitzis, 2011. Temperature, entropy and heat capacity of a non-newtonian black hole resulting from a yukawa correction to the metric. Astrophys Space Sci., 337: 693-702.

Haranas, I. and I. Gkigkitzis, 2013a. Bekestein bound of information number $n$ and its relation to cosmological parameters in a universe with and without cosmological constant. Mod. Phys. Lett. A, 28: 19-19.

Haranas, I. and I. Gkigkitzis, 2013b. Entropic gravity resulting from a Yukawa type of correction to the metric for a solar mass black hole. Astrophys Space Sci., 347: 77-82. DOI: 10.1007/s10509013-1492-4

Henry, R.C., 2000. Kretschmann scalar for a kerrnewman black hole. Astrophysical. J. E, 353: 535350. DOI: $10.1086 / 308819$

Horodecki, M., J. Oppenheim and A. Winter, 2005. Partial quantum information. Nature, 436: 673-676. DOI: 10.1038/nature03909

Misner, C.W. and J.A. Wheeler, 1973. Gravitation. 1st Edn., Freeman, New York, ISBN-10: 0716703440, pp: 1279.

Rotundo, G. and M. Ausloos, 2013. Complex-valued information entropy measure for networks with directed links (digraphs). Application to citations by community agents with opposite opinions. Eur. Phys. J. B, 86: 1-10. DOI: 10.1140/epjb/e201330985-6

Spallicci, A.D.A.M., 1991. The fifth force in the schwarzschild metric and in the field equations: The concept of parageodesic motions. Annalen der Physik, 503: 365-368. DOI: 10.1002/andp.19915030509

Stephani, H., 1990. General Relativity: An Introduction to the Theory of the Gravitational Field. 2nd Edn, Cambridge, University Press, pp: 124.

Zhang, B., Q.Y. Cai, M.S. Zhan and L. You, 2011. An interpretation for the entropy of a black hole. General Relat. Gravit., 43: 797-804. DOI: 10.1007/s10714-010-1097-y

Zwerger, W., 1985. Dynamical interpretation of a classical complex free energy. J. Phys. A, 18: 20792085. DOI: 10.1088/0305-4470/18/11/028 